\title{
Third Places for Health Promotion with Older Adults: Using the Consolidated Framework for Implementation Research to Enhance Program Implementation and Evaluation
}

Mary E. Northridge, Susan S. Kum, Bibhas Chakraborty, Ariel Port Greenblatt, Stephen E. Marshall, Hua Wang, Carol Kunzel, and Sara S. Metcalf

\begin{abstract}
This study extends the concept of third places to include community sites where older adults gather, often for meals or companionship. The Consolidated Framework for Implementation Research guided program implementation and evaluation. Depending upon health promotion program needs, the physical infrastructure of a site is important, but a supportive director (champion) can often overcome identified deficits. Senior centers may be locally classified into four types based upon eligibility requirements of residents in affiliated housing and services offered. Participants who attend these centers differ in important ways across types by most sociodemographic as well as certain health and health care characteristics.
\end{abstract}

KEYWORDS Community-based programs, Health promotion, Health service delivery, Older adults, Oral bealth

\section{INTRODUCTION}

Nearly three decades ago, Oldenburg introduced the concept of "great good places," also termed "third places," as public places on neutral ground where people may gather, enjoy the company of others, and interact. ${ }^{1,2}$ He contrasted the voluntary, informal, and anticipated gatherings that characterize third places to those that occur among family members in first places (home) and among employees in second places (work). In his view, beer gardens, main streets, pubs, cafés, coffeehouses, post offices, and other third places are "hangouts" that form the heart of a community. 1,2

Northridge and Greenblatt are with the Department of Epidemiology and Health Promotion, New York University College of Dentistry, 433 First Avenue, Room 726, New York, NY 10010, USA; Northridge, Kum, and Metcalf are with the Department of Geography, The State University of New York at Buffalo, 105 Wilkeson Quad, Buffalo, NY, USA; Northridge and Kunzel are with the Department of Sociomedical Sciences, Columbia University Mailman School of Public Health, 722 West 168th Street, New York, NY, USA; Northridge, Marshall, and Kunzel are with the Columbia University College of Dental Medicine, 630 West 168th Street, New York, NY, USA; Chakraborty is with the Duke-National University of Singapore (Duke-NUS) Medical School, Centre for Quantitative Medicine, 20 College Road, Level 6, Academia, Singapore; Wang is with the Department of Communication, The State University of New York at Buffalo, 359 Baldy Hall, Buffalo, NY, USA.

Correspondence: Mary E. Northridge, Department of Epidemiology and Health Promotion, New York University College of Dentistry, 433 First Avenue, Room 726, New York, NY 10010, USA. (E-mail: men6@nyu.edu)

Mary E. Northridge and Susan S. Kum share co-first authorship 
The potential health-promoting effects of third places have received scientific attention and preliminary study. ${ }^{3,4}$ Glover and Parry broadened the definition of third places by arguing that Gilda's Club of Greater Toronto deserves this distinction, even as it is a place apart from home and hospital (rather than work) and represents a somewhat exclusive rather than public gathering place for people living with cancer. ${ }^{5}$ Hooper et al. also extended the concept of third places by demonstrating that creating third places from everyday environments is likely to start in childhood, potentially carrying on, with varying degrees of success, into adult lives. ${ }^{6}$ Finally, Northridge et al. posited that online sites might serve as third places for older adults seeking health promotion and disease management information in Harlem, New York. ${ }^{7}$ This may be especially important as deprived socioeconomic areas have been found to be lacking in the type of third-place amenities that might be supportive of health through encouraging contact between people. ${ }^{8}$

From the outset, the ElderSmile program of the Columbia University College of Dental Medicine elected to conduct its community-based oral health outreach program activities in community sites where older adults gather, i.e., third places. ${ }^{9}$ The foremost reason that the ElderSmile program elected to focus on creating a network of prevention centers in third places was to access a population of older adults that was not centrally interested in obtaining oral health care for painful conditions, that is, to intervene before disease is severe. ${ }^{10}$ Given ongoing partnership with the prevention centers over time, both older adults who received services and center directors, who value the ElderSmile program, help recruit additional participants for subsequent oral health screenings and other program activities, now including diabetes and hypertension screenings. ${ }^{9-11}$

While initially the ElderSmile program considered a variety of third places for prevention activities, including barber shops and other community amenities, it became clear that both social and physical infrastructure were necessary to operate the program. In thinking through the intervention and evaluation priorities for this ongoing community-based project, the Consolidated Framework for Implementation Research (CFIR) proved useful, as it provides a menu of constructs that have been associated with effective implementation and may be used in a range of applications. ${ }^{12,13}$ The five major domains of the CFIR are (1) the intervention characteristics, (2) the outer setting, (3) the inner setting, (4) the characteristics of the individuals involved, and (5) the process by which implementation is accomplished. Eight constructs are related to the intervention (e.g., adaptability and costs), 4 constructs are related to the outer setting (e.g., patient needs and external policy), 12 constructs are related to the inner setting (e.g., social networks and leadership engagement), 5 constructs are related to the characteristics of individuals (e.g., self-efficacy and stage of change), and 8 constructs are related to the process (e.g., planning and reflecting). See the Appendix for a full listing of the 37 CFIR constructs under 5 domains.

Guided by the CFIR, the aims of this paper are to (1) detail the rationale for why certain third places were selected for participation in the ElderSmile network, (2) introduce a locally relevant method for categorizing these third places into center types that may prove transferable or adaptable for other locales, and (3) describe the characteristics of the participants who were screened at each center type. Throughout its operation, ElderSmile has incorporated a spatial approach for addressing socioeconomic disparities in health for older adults and planning community-based health promotion activities. ${ }^{14-17}$ A fourth aim of this communication is thus to extend this tradition by incorporating an exploratory spatial approach to evaluate program implementation that may prove insightful for other programs that serve older adults. In the remainder of this paper, the corresponding CFIR constructs are identified in parentheses after characteristics or processes involved in the 
intervention. For example, the ElderSmile program (intervention) is the focus of this research.

\section{METHODS}

Throughout the course of conducting this study, all Columbia University, New York University, and University at Buffalo institutional review board and Health Insurance Portability and Accountability Act safeguards were followed.

\section{Selection of Third Places for the ElderSmile Network}

The formation of the ElderSmile network of third places (network), also referred to as prevention centers, emerged because the foremost goal of the program, namely, to enhance the oral health of older adults in northern Manhattan, coincided with the mission of the Columbia University College of Dental Medicine, that is, to improve the oral health of its surrounding neighborhoods (external setting). In order to have the greatest community-level impact, the program founders (leaders) aimed to include a group of geographically diverse locations to ensure that the prevention centers were conveniently located within close proximity to most of the places where older adults (the individuals involved) lived. Prerequisites for site selection (the inner setting) included the following social and physical infrastructure: (1) the location needed to attract a sufficient number of older adults each day (approximately 25 or more), (2) older adults ought to congregate at the prevention center for an extended period of time (i.e., over $2 \mathrm{~h}$ ), and (3) the site mission and director should express an interest in improving the health and quality of life of older adults. In order to conduct a successful outreach event, a prevention center also needed to have sufficient space and ideally, dedicated rooms in which to host the interactive educational slide deck presentation and perform screenings for oral disease, diabetes, and hypertension. Without sufficient space for these educational and screening activities, the outreach event flow was often hindered, and as a result, fewer participants were able to participate. Finally, the dedicated rooms needed to be well-lit and wellventilated and provide access to electrical outlets and running water. Although few sites met all of these requirements, the ElderSmile outreach team members (formally appointed internal implementation leaders) were creative and skilled in ensuring that less than ideal physical infrastructure was nonetheless adequate to host prevention activities.

The single most important factor in determining the success of a prevention center event was the support and organization of its director (external change agent). As a result, the ElderSmile outreach team deliberately sought to develop rapport with prevention center directors toward ensuring the sustainability of the program. Time and again, our experience was that an enthusiastic and engaged center director (champion) could overcome any physical infrastructure deficits, and conversely, a disinterested and uncommitted center director could undermine even ideal physical space. Since senior centers and locations where older adults congregate were not used for the sole purpose of health screenings, other recreational activities, such as domino games and walking outside, often occurred simultaneously with outreach events. A supportive and effective director would encourage older adults to participate in ElderSmile outreach events and request that other competing activities be suspended during the educational slide deck presentation, at a minimum.

In summary, ElderSmile events held in locations with supportive leaders resulted in successful outreach activities with high participation rates, while ElderSmile events held in locations with disinterested or absentee leaders resulted in unsuccessful outreach activities with low participation rates. By way of illustration, a particularly frustrating situation 
occurred when the outreach team arrived at a prevention center only to discover that most of the center regulars left on a bus trip to Atlantic City, NJ. As a result, the outreach event was canceled, even though the ElderSmile outreach staff had confirmed the date multiple times with the center director.

\section{Locally Relevant Method for Classifying Third Places}

Resources from authoritative governmental and advocacy groups for the aging were consulted in developing a locally relevant method for classifying third places for the ElderSmile network of prevention centers. ${ }^{18}, 19$ This classification system was developed by the co-first author (S.S.K.) and verified by ElderSmile team members who also serve as co-authors. The four center types in the resulting classification system are (1) Naturally Occurring Retirement Community (NORC), (2) US Department of Housing and Urban Development Section 202 Supportive Housing for the Elderly Program (Section 202), (3) New York City Housing Authority (NYCHA), and (4) community senior or resource center (Community Center). Each of these four center types is further described in the following section.

\section{Measures}

The individual-level data for this study were provided by self-report of participants or collected by staff and dentists of the ElderSmile program via intake interviews, clinical dental assessments, and measurement of glycosylated hemoglobin (HbA1c) and blood pressure (BP). Details of the ElderSmile clinical program and the primarycare screening enhancements are provided elsewhere. ${ }^{9-11,20-22}$

Center Type As previously mentioned, a locally relevant method for classifying third places for older adults in urban settings was developed consisting of four center types. The first center type is NORC, i.e., a housing complex or neighborhood with residential dwellings that were not purposefully planned for older adults and do not restrict admission to older adults, but which house high concentrations of residents aged 60 years and older of low to moderate income, as residents have aged in place. New York State supports the following two NORC programs: (1) NORC Supportive Service Program, for housing complexes or apartment buildings that were built with government assistance, with $50 \%$ of the units with an elderly occupant or 2500 residents aged 60 years or older, and (2) Neighborhood NORC, for a residential dwelling or group of residential dwellings in a geographically defined area with no more than 2000 people who are aged 60 years or older residing in at least $40 \%$ of the units, made up of low-rise buildings (six stories or less), single or multi-family homes not originally built for older adults. ${ }^{23}$ NORCs provide support services such as health and wellness activities, socialization events, home-delivered meals, and transportation. In New York City, NORC programs are public-private partnerships with the New York City Department for the Aging, the United Hospital Fund, housing entities, community service providers, and NORC residents. ${ }^{24}$

The second center type is Section 202, i.e., a US Department of Housing and Urban Development program that offers affordable housing opportunities specifically for low- or moderate-income older adults. This program provides rent subsidies and capital advances, i.e., interest-free grants or loans, for constructing or rehabilitating housing structures. To be eligible for Section 202 housing, one person in the household must be 62 years old at the initial occupancy of the housing unit. Support services such as congregate meals, cleaning, and transportation are provided to facilitate independent living. ${ }^{25}$ 
The third center type is NYCHA, i.e., a public housing authority that offers affordable housing to low- and moderate-income residents in the five boroughs of New York City through the Conventional Public Housing Program or Section 8 Leased Housing Program. Housing developments and buildings exclusively for residents aged 62 years and older are available. Senior centers are located within New York City Housing Authority developments that provide recreational activities and meals for senior residents and community members in the surrounding neighborhoods. These senior centers are operated by or in partnership with New York City Housing Authority, New York City Department for the Aging, and community organizations. ${ }^{26}$

Finally, a senior center or resource center is a place in the community where older adults can socially interact with others and receive support services, which together comprise the fourth center type, namely, Community Center. Senior centers provide a range of services to adults typically aged 60 and older, notably health and wellness promotion and education. While senior centers vary in physical setting and size, populations served, services offered, and funding sources, a principal service offered by all senior centers is congregate meals-at least one meal a day, 5 days a week. ${ }^{27}$ Resource centers function similarly to senior centers but may not provide services or congregate meals on a regular basis.

Sociodemographic, Health, and Health Care Characteristics Sociodemographic information on ElderSmile participants was obtained by questionnaire in English or Spanish, according to participant preferences. The data gathered included age, gender, race, Hispanic ethnicity, language spoken at home, place of birth, highest level of education attained, and home address. For the purposes of the analyses presented here, participants were categorized by age as 50-64, 65-74, or $75+$ years. Race/ethnicity was categorized as non-Hispanic white, non-Hispanic black, Hispanic, and other. Place of birth was categorized as mainland USA, Dominican Republic, Puerto Rico, and other. The highest level of education attained was categorized as primary school, high school, or some college or more.

Health and health care information were collected by self-report or clinical assessments. Self-reported smoking status was characterized as never smoked, former smoker, or current smoker. Participants were asked if they had medical insurance (yes, no), and if so, what type (Medicaid, Medicare, or private); if they had dental insurance (yes, no), and if so, what type (Medicaid or private); and the times since their last medical and dental visits $(<1,1-3$, and $>3$ years).

Specifically, with regard to oral health, participants assessed the status of their teeth and gums as excellent, good, fair, or poor. A faculty dentist performed a screening assessment on older adults who agreed to participate. Participants were examined for the number and condition of their teeth, and the number of teeth present was categorized as edentulous $(n=0)$, limited function $(n=1-19)$, and functional dentition $(n=20-28) .^{28}$

Glycemic status (normoglycemic, pre-diabetes, or diabetes) as assessed by HbA1c was measured by a point-of-care test using capillary (finger stick) blood via a DCA Vantage Analyzer. ${ }^{29}$ Cut-points for measured HbA1c were as follows: normoglycemic $=\mathrm{HbA} 1 \mathrm{c}<5.6 \%$, pre-diabetes $=\mathrm{HbA} 1 \mathrm{c}$ between 5.7 and $6.4 \%$, and diabetes $=\mathrm{HbA} 1 \mathrm{c}>6.5 \% \cdot{ }^{30}$ For participants with previously diagnosed diabetes, an HbA1c of $7.0 \%$ or higher was considered as poor glycemic control, and an HbA1c of less than $7.0 \%$ was considered as acceptable glycemic control. 
Finally, both systolic BP (SBP) and diastolic BP (DBP) were measured using an Omron blood pressure monitor. ${ }^{31}$ The cut-points for blood pressure status were as follows: (1) normal = SBP of $120 \mathrm{mmHg}$ or less and DBP of $80 \mathrm{mmHg}$ or less, (2) prehypertension = SBP between 120 and $139 \mathrm{mmHg}$ or DBP between 80 and $89 \mathrm{mmHg}$, and (3) hypertension $=$ SBP of $140 \mathrm{mmHg}$ or more or DBP of $90 \mathrm{mmHg}$ or more. ${ }^{32}$

\section{Analytic Approach}

Descriptive statistics for self-reported sociodemographic, health, and health care characteristics of participants at ElderSmile outreach events held between July 2006 and October 2013 by center type were computed in $R .^{33}$ Tests for the differences among participants across center types were conducted using the chi-squared test. ${ }^{34}$

Addresses of third places and ElderSmile participants with addresses in Manhattan and the Bronx were processed with the Geosupport Desktop Edition. ${ }^{35}$ Maps were generated with ArcGIS Desktop. ${ }^{36}$ The residential locations of participants were recorded at intake into the ElderSmile program, and the street addresses of centers were supplied by the ElderSmile program staff and verified by the co-first author (S.S.K.) via public data sources. Only participants who resided in Manhattan or the Bronx and furnished addresses without errors were georeferenced.

\section{RESULTS}

There were 2026 electronic records available for analysis of participants who attended ElderSmile outreach events between July 2006 and October 2013. Of these, 182 records with self-reported residential addresses that could not be georeferenced to locations in Manhattan or the Bronx, and 21 records from two ElderSmile events where the center type could not be classified with certainty, were excluded. The results presented next are based on 1823 records or $90.0 \%$ of the original 2026 records.

\section{Characteristics of ElderSmile Participants Overall}

The largest number and percentage of participants were seen at Community Centers $(n=$ 711 or $39.0 \%$ ), followed by NYCHA, Section 202, and NORC center types (Table 1). Most of the participants were female $(72.6 \%)$ and Hispanic $(57.2 \%)$. Approximately equal percentages were aged $65-74$ years $(41.8 \%)$ and greater than or equal to aged 75 years $(41.0 \%)$, even as a fair percentage was less than aged 65 years $(17.3 \%)$. While most of the participants spoke English as their primary language $(53.5 \%)$, almost as many spoke Spanish as their primary language (42.9\%). Most were born outside of the mainland USA $(60.5 \%)$ and were covered by Medicaid $(51.7 \%)$. Over a third $(37.8 \%)$ reported their highest educational attainment as primary school. Most had never smoked $(58.1 \%)$, had not visited a doctor $(53.6 \%)$ or a dentist $(51.5 \%)$ in the last year, rated their oral health as fair or poor $(59.9 \%)$, and were edentulous or had limited function dentition $(64.3 \%)$. The burden of chronic disease among participants was high, with $43.4 \%$ self-reporting diabetes and $64.1 \%$ with glycemic status in the pre-diabetes or diabetes range and $70.1 \%$ self-reporting hypertension and $80.5 \%$ with blood pressure measurements in the pre-hypertension or high range.

\section{Characteristics of ElderSmile Participants by Center Type}

There were significant differences by center type among ElderSmile participants by all of the sociodemographic characteristics examined (Table 1). For instance, a higher percentage of men $(31.8 \%)$ attended ElderSmile events at Community Centers than other center types, and a higher percentage of Hispanics $(65.1 \%)$ 
TABLE 1 Sociodemographic, health, and health care characteristics of ElderSmile participants by center type, New York, NY, 2006-2013

\begin{tabular}{|c|c|c|c|c|c|c|}
\hline Characteristic & $\begin{array}{l}\text { Community } \\
\text { Center }\end{array}$ & NORC & NYCHA & Section 202 & Overall & $\begin{array}{l}p \\
\text { Value }^{\mathrm{b}}\end{array}$ \\
\hline & $\left(n^{\mathrm{a}}=711\right)$ & $\begin{array}{l}\left(n^{\mathrm{a}}=\right. \\
125)\end{array}$ & $\begin{array}{l}\left(n^{\mathrm{a}}=\right. \\
653)\end{array}$ & $\left(n^{\mathrm{a}}=334\right)$ & $\begin{array}{l}\left(n^{\mathrm{a}}=\right. \\
1823)\end{array}$ & \\
\hline Gender, \% & & & & & & $<0.01$ \\
\hline Female & 68.2 & 73.2 & 78.6 & 70.4 & 72.6 & \\
\hline Male & 31.8 & 26.8 & 21.4 & 29.6 & 27.4 & \\
\hline Race/ethnicity, \% & & & & & & $<0.01$ \\
\hline Hispanic & 53.5 & 43.3 & 65.1 & 54.7 & 57.2 & \\
\hline Non-Hispanic white & 17.3 & 25.0 & 5.0 & 4.8 & 11.2 & \\
\hline Non-Hispanic black & 26.0 & 17.5 & 28.0 & 37.6 & 28.2 & \\
\hline Other & 3.2 & 14.2 & 1.9 & 2.9 & 3.5 & \\
\hline Age group, \% & & & & & & $<0.01$ \\
\hline$<65$ years & 18.7 & 11.6 & 20.3 & 10.2 & 17.3 & \\
\hline 65-74 years & 39.2 & 44.6 & 43.3 & 43.3 & 41.8 & \\
\hline$\geq 75$ years & 42.2 & 43.8 & 36.4 & 46.6 & 41.0 & \\
\hline Primary language, \% & & & & & & $<0.01$ \\
\hline English & 54.9 & 56.2 & 48.9 & 58.8 & 53.5 & \\
\hline Spanish & 41.2 & 32.2 & 48.9 & 38.8 & 42.9 & \\
\hline Other & 3.9 & 11.6 & 2.2 & 2.5 & 3.6 & \\
\hline Place of birth, \% & & & & & & $<0.01$ \\
\hline Mainland USA & 40.2 & 37.5 & 35.8 & 45.7 & 39.5 & \\
\hline Puerto Rico & 19.9 & 12.5 & 28.7 & 21.1 & 22.8 & \\
\hline Dominican Republic & 13.8 & 18.3 & 22.2 & 21.5 & 18.5 & \\
\hline Other & 26.1 & 31.7 & 13.3 & 11.7 & 19.2 & \\
\hline Medicaid coverage, $\%$ & & & & & & $<0.01$ \\
\hline Yes & 48.8 & 25.2 & 53.4 & 64.1 & 51.7 & \\
\hline No & 51.2 & 74.8 & 46.6 & 35.9 & 48.3 & \\
\hline Highest education, \% & & & & & & $<0.01$ \\
\hline Primary & 35.5 & 22.5 & 39.6 & 45.2 & 37.8 & \\
\hline High school & 33.3 & 22.5 & 38.9 & 36.9 & 35.2 & \\
\hline Some college or more & 31.1 & 55.0 & 21.5 & 17.9 & 26.9 & \\
\hline Smoking status, \% & & & & & & $<0.01$ \\
\hline Never smoked & 62.3 & 59.6 & 55.3 & 53.6 & 58.1 & \\
\hline Former smoker & 29.2 & 35.1 & 29.7 & 32.1 & 30.3 & \\
\hline Current smoker & 8.5 & 5.3 & 15.0 & 14.3 & 11.6 & \\
\hline $\begin{array}{l}\text { Time since last visit to a } \\
\text { doctor, } \%\end{array}$ & & & & & & 0.09 \\
\hline$<1$ year & 45.1 & 41.2 & 47.2 & 49.7 & 46.4 & \\
\hline $1-3$ years & 45.9 & 51.3 & 44.1 & 37.5 & 44.1 & \\
\hline$>3$ years & 9.1 & 7.6 & 8.8 & 12.8 & 9.5 & \\
\hline $\begin{array}{l}\text { Time since last visit to a } \\
\text { dentist, \% }\end{array}$ & & & & & & 0.02 \\
\hline$<1$ year & 48.7 & 59.8 & 48.2 & 44.3 & 48.5 & \\
\hline $1-3$ years & 32.1 & 29.5 & 31.6 & 29.7 & 31.3 & \\
\hline$>3$ years & 19.2 & 10.7 & 20.2 & 26.0 & 20.2 & \\
\hline Self-rated oral health, \% & & & & & & 0.33 \\
\hline Good or better & 42.4 & 39.4 & 40.0 & 35.5 & 40.1 & \\
\hline Fair & 37.5 & 32.3 & 37.5 & 40.5 & 37.7 & \\
\hline Poor & 20.1 & 28.3 & 22.5 & 24.0 & 22.2 & \\
\hline
\end{tabular}


TABLE 1 Continued

\begin{tabular}{|c|c|c|c|c|c|c|}
\hline Characteristic & $\begin{array}{l}\text { Community } \\
\text { Center }\end{array}$ & NORC & NYCHA & Section 202 & Overall & $\begin{array}{l}p \\
\text { Value }^{\mathrm{b}}\end{array}$ \\
\hline & $\left(n^{\mathrm{a}}=711\right)$ & $\begin{array}{l}\left(n^{\mathrm{a}}=\right. \\
125)\end{array}$ & $\begin{array}{l}\left(n^{\mathrm{a}}=\right. \\
653)\end{array}$ & $\left(n^{\mathrm{a}}=334\right)$ & $\begin{array}{l}\left(n^{\mathrm{a}}=\right. \\
1823)\end{array}$ & \\
\hline $\begin{array}{l}\text { Dentition status ( } n \text { teeth), } \\
\quad \%\end{array}$ & & & & & & $<0.01$ \\
\hline Edentulous $(n=0)$ & 16.1 & 13.0 & 22.9 & 24.3 & 19.7 & \\
\hline Limited $(n=1-19)$ & 42.9 & 40.0 & 46.5 & 47.1 & 44.6 & \\
\hline Functional ( $n=20-28)$ & 41.0 & 47.0 & 30.6 & 28.6 & 35.7 & \\
\hline Self-reported diabetes, \% & & & & & & 0.52 \\
\hline Yes & 44.4 & 36.7 & 41.5 & 47.2 & 43.4 & \\
\hline No & 55.6 & 63.3 & 58.5 & 52.8 & 56.6 & \\
\hline $\begin{array}{l}\text { Self-reported } \\
\text { hypertension, \% }\end{array}$ & & & & & & 0.19 \\
\hline Yes & 74.0 & 64.3 & 66.5 & 70.6 & 70.1 & \\
\hline No & 26.0 & 35.7 & 33.5 & 29.4 & 29.9 & \\
\hline Glycemic status, \% & & & & & & 0.82 \\
\hline Normal & 36.2 & 36.6 & 37.9 & 30.8 & 35.9 & \\
\hline Pre-diabetes & 39.3 & 36.6 & 34.9 & 40.0 & 37.5 & \\
\hline Diabetes & 24.5 & 26.8 & 27.2 & 29.2 & 26.6 & \\
\hline Blood pressure status, \% & & & & & & 0.29 \\
\hline Normal & 15.5 & 20.9 & 21.6 & 23.2 & 19.4 & \\
\hline Pre-hypertension & 39.6 & 46.5 & 36.5 & 35.2 & 38.0 & \\
\hline High blood pressure & 44.9 & 32.6 & 41.9 & 41.5 & 42.5 & \\
\hline
\end{tabular}

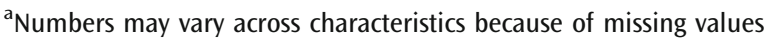

${ }^{\mathrm{b}} p$ Values correspond to the testing of differences among participants across center types using the chisquared test

and adults aged 65 years or younger $(20.3 \%)$ attended ElderSmile events at NYCHA sites than other center types. Further, more participants at NORC sites spoke a primary language other than English or Spanish $(11.6 \%)$ or attended some college or more $(55.0 \%)$ than at other center types, while more participants at Section 202 sites were born in the mainland USA $(45.7 \%)$ than at other sites.

There were fewer significant differences by health and health care characteristics among ElderSmile participants by center type, perhaps because of the overall high burden of chronic conditions in this population. Nonetheless, more participants at Section 202 centers than other center types had Medicaid coverage $(64.1 \%)$, more participants at Community Centers than other center types never smoked $(62.3 \%)$, and more participants at NORCs had visited a dentist in the last year $(59.8 \%)$ and had functional dentition $(47.0 \%)$ than at other center types.

\section{Residential Locations of ElderSmile Participants by Center Type}

Toward understanding where the ElderSmile participants lived and how far they travelled to attend outreach events, the residential locations of participants were mapped and colored to correspond to the center type where they attended an outreach event (Fig. 1). Most of the ElderSmile participants lived in northern Manhattan, even as they also travelled from the Bronx and locations throughout Manhattan. Clustering of participants by the center type 


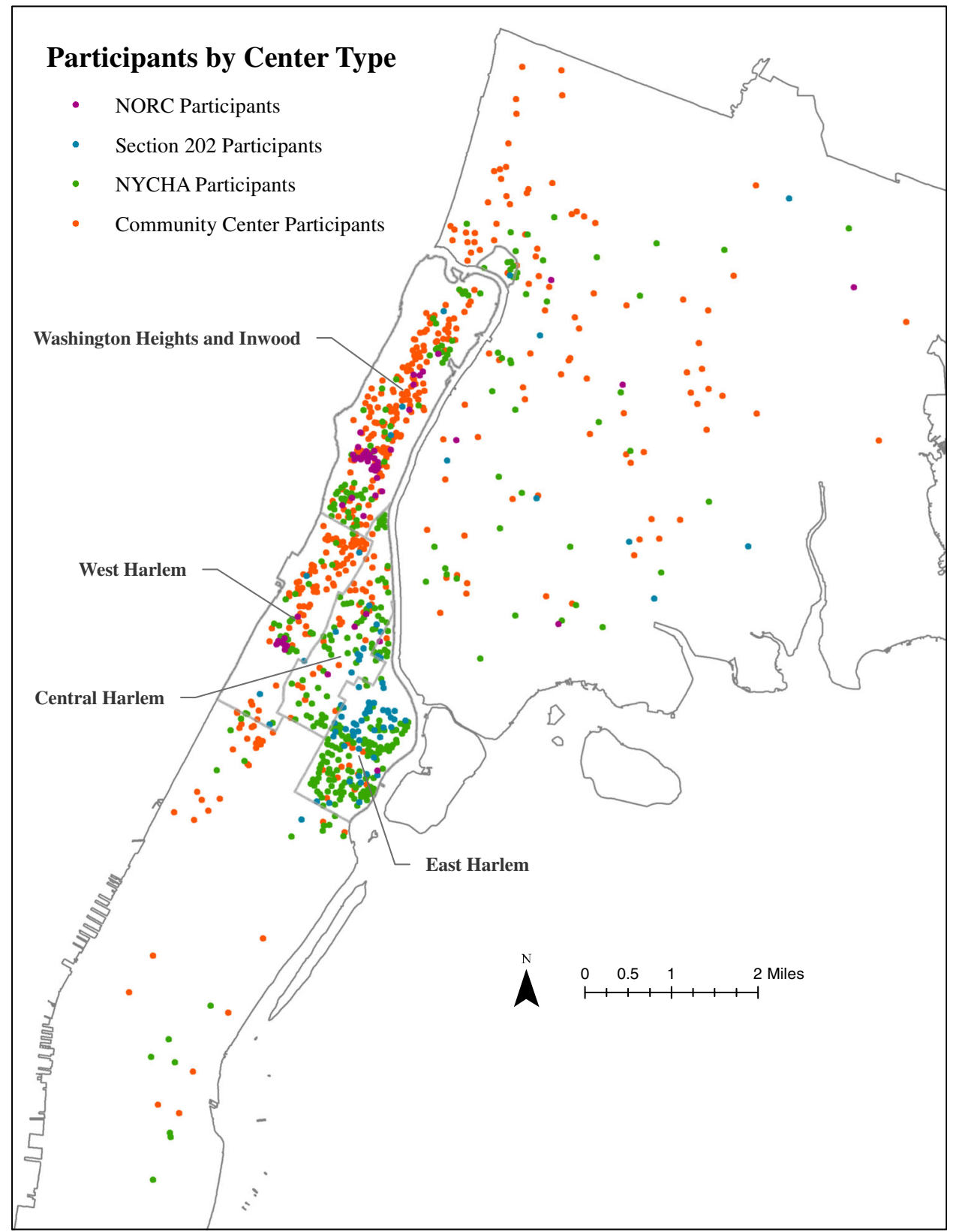

FIG. 1 Residential locations of participants, colored to correspond to the center type where they attended an ElderSmile outreach event, New York, NY, 2006-2013.

where they attended an outreach event is more concentrated for certain center types than others; e.g., the NYCHA centers are concentrated in East Harlem.

\section{Residential Locations of ElderSmile Participants Who} Attended Outreach Events at Community Centers

The residential locations of ElderSmile participants who attended outreach events at a Community Center and the straight-line paths from their residential locations to the 


\section{Community Center}

- Centers $(n=17)$

\section{Participants}

- $1(n=383)$

- $2(\mathrm{n}=81)$

- 3-10 (n=35)

$11+(n=1)$

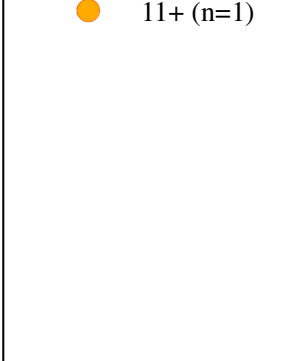




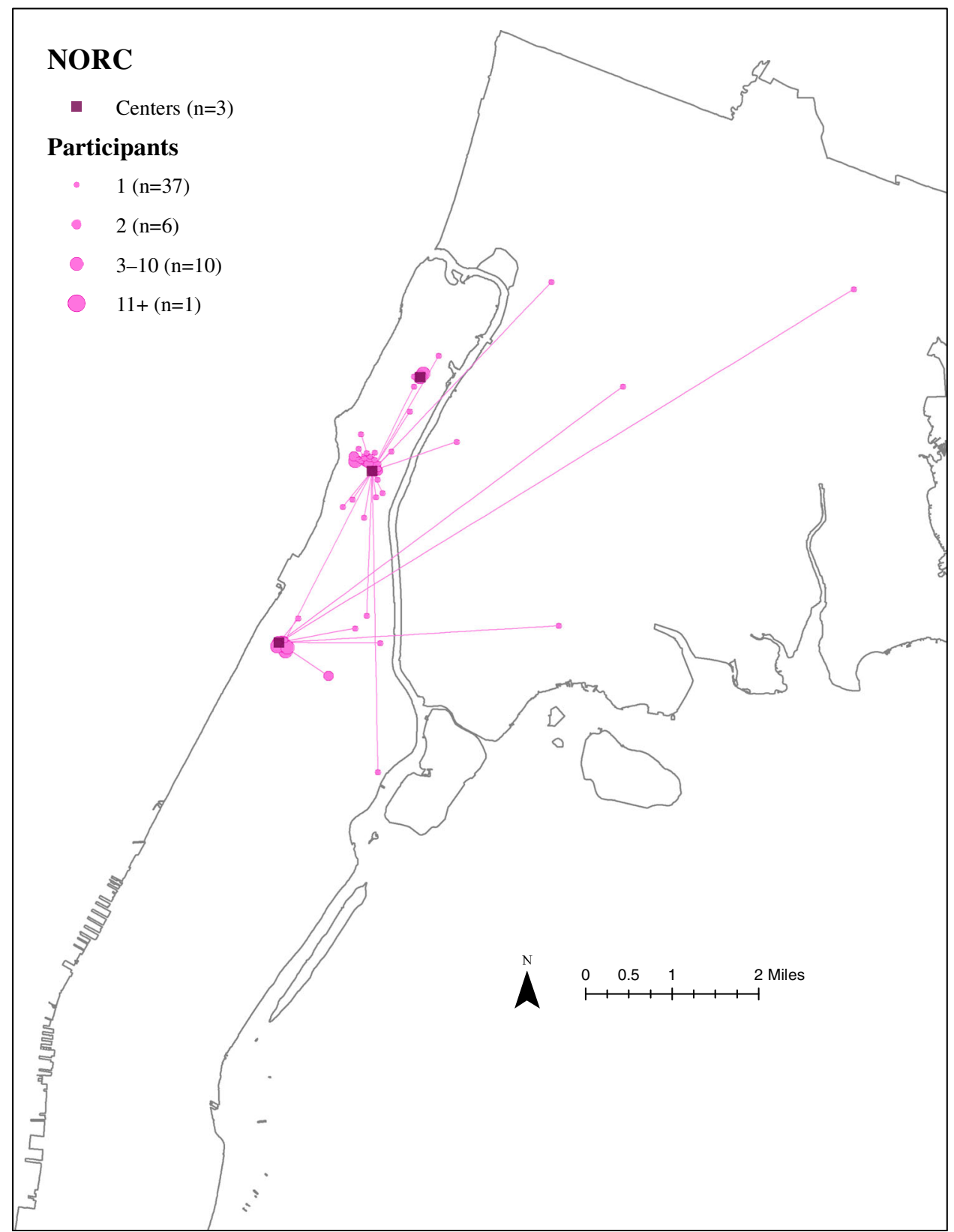

FIG. 3 Residential locations of ElderSmile participants who attended outreach events at a Naturally Occurring Retirement Community (NORC) center and the straight-line paths from their residential locations to the NORCS where they attended these events, 2006-2013.

relatively far from their own locations and had the largest number of participants at a unique residential address $(n=383)$ of any center type.

\section{Residential Locations of ElderSmile Participants Who}

\section{Attended Outreach Events at NORCs}

The residential locations of ElderSmile participants who attended outreach events at a NORC and the straight-line paths from their residential locations to the NORCs where 


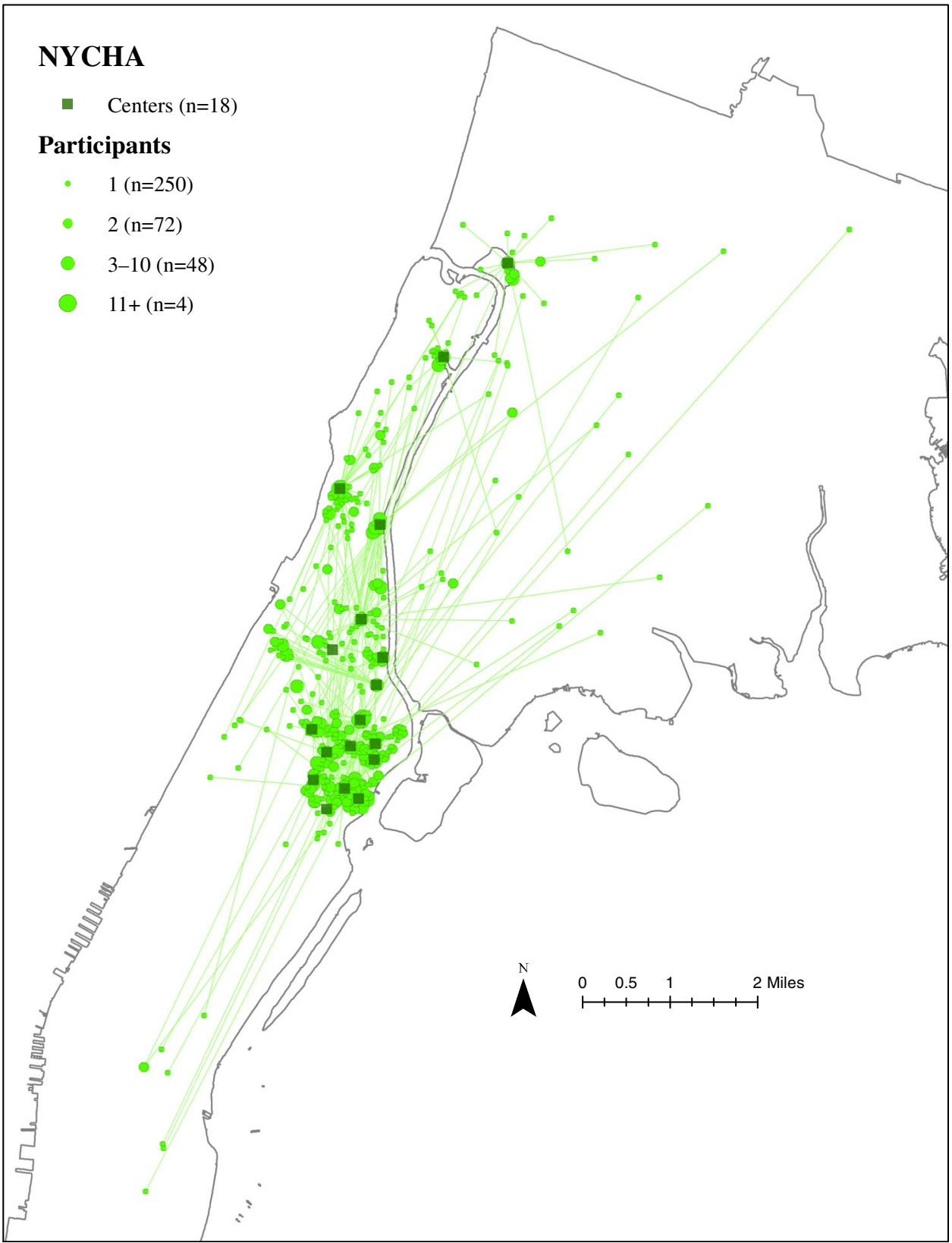

FIG. 4 Residential locations of ElderSmile participants who attended outreach events at a New York City Housing Authority (NYCHA) center and the straight-line paths from their residential locations to the NYCHA centers where they attended these events, 2006-2013.

they attended these events are depicted in Fig. 3. Note that the majority of the participants who attended an ElderSmile event at one of the three NORCs lived in close proximity to the sites. Nonetheless, the patterns reflect the operations and missions of the NORCs, the northernmost NORC primarily services residents at three affiliated housing complexes in close proximity to the site, 


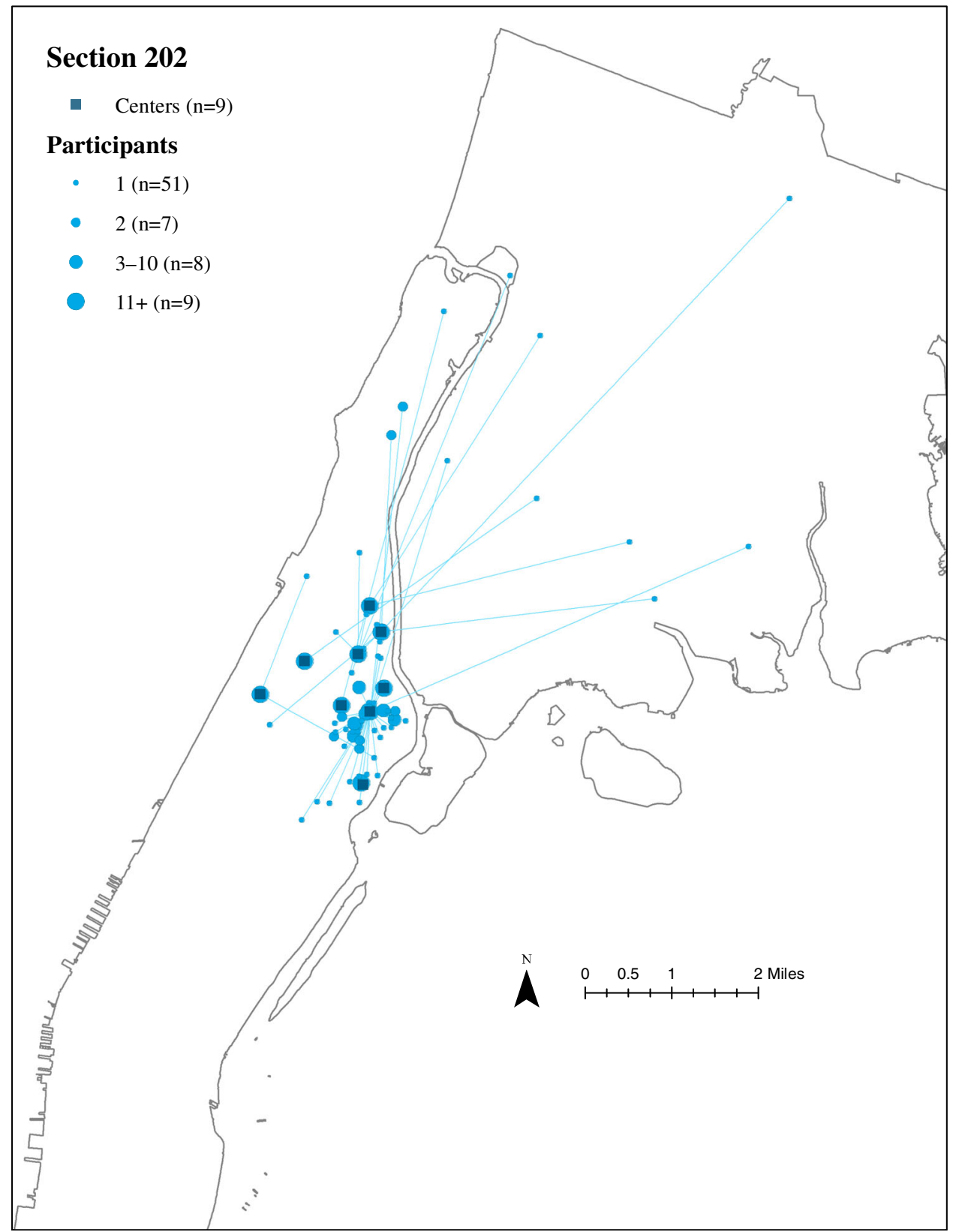

FIG. 5 Residential locations of ElderSmile participants who attended outreach events at a US Department of Housing and Urban Development Section 202 Supportive Housing for the Elderly Program (Section 202) center and the straight-line paths from their residential locations to the Section 202 centers where they attended these events, 2006-2013.

the NORC in the center of the other two NORCs is a neighborhood NORC and services residents who live both nearby and farther away from the site, and the southernmost NORC partners with an organization that provides services in six affiliated housing complexes in a defined catchment area. 


\section{Residential Locations of ElderSmile Participants Who Attended Outreach Events at NYCHA Centers}

The residential locations of ElderSmile participants who attended outreach events at a NYCHA center and the straight-line paths from their residential locations to the NYCHA centers where they attended these events are depicted in Fig. 4. Note that there are more NYCHA centers $(n=18)$ than any other center type. NYCHA center services are open to both residents who reside at the sites as well as older adults in nearby and outlying communities.

\section{Residential Locations of ElderSmile Participants Who Attended Outreach Events at Section 202 Centers}

The residential locations of ElderSmile participants who attended outreach events at a Section 202 center and the straight-line paths from their residential locations to the Section 202 centers where they attended these events are depicted in Fig. 5. In contrast to the Community Centers and NYCHA centers, Section 202 centers tend to draw participants from the housing complexes with which they are affiliated.

\section{DISCUSSION}

A locally relevant method for categorizing third places introduced here proved useful in understanding differences among ElderSmile participants who attended outreach events by the center types they visited. Certain findings confirmed the validity of the locally relevant classification method developed as part of this research. For instance, the highest percentages of participants aged 75 years and older attended outreach events in Section 202 centers $(46.6 \%)$ and NORCs $(43.8 \%)$, and both of the housing programs with which these center types are affiliated have older age eligibility requirements. Further, the highest percentages of participants with Medicaid coverage attended outreach events in Section 202 centers $(64.1 \%)$ and NYCHA centers $(53.4 \%)$, and both of the housing programs with which these center types are affiliated have income eligibility requirements.

On the other hand, this classification system revealed unanticipated differences among participants by the center types they visited that may be useful in planning future outreach events. Note that the highest percentage of participants who had not visited a dentist for more than 3 years $(26.0 \%)$ and were edentulous $(24.3 \%)$ or had limited function dentition $(47.1 \%)$ attended outreach events at Section 202 centers. Older adults may not be aware that oral health visits are important regardless of the number of teeth they retain (reflecting).

The exploratory spatial analyses presented here add value beyond the tabulated differences among ElderSmile participants by characteristics across center types. Clearly, certain centers and types drew larger numbers of participants and had larger catchment areas than others. It is also evident that participants are not necessarily attending outreach events at the centers located closest to their residential locations. Next steps include conducting analyses using the transport network in New York City (including both train and bus routes) to estimate travel time distances for participants attending ElderSmile outreach events (planning).

\section{Strengths and Limitations of the Study}

A major strength of this study is that these analyses were conducted in close collaboration with the ElderSmile founders, faculty dentists, and staff (implementation leaders) who had firsthand knowledge of the included centers and their directors (champions and change agents). Further, the geographers who are leaders and members of the research team possess both the expertise to conduct meaningful spatial analyses and the 
skills to create accessible maps to guide program implementation and evaluation (executing). Notable limitations include that we were unable to account for repeat participants at the centers and were missing essential information for 352 records that were thus not included in the analyses reported here. Further, while the classification system used in this study was locally relevant for northern Manhattan, it would need to be adapted to the context of other geographic areas, where health promotion programs are implemented for older adults. Nonetheless, there may be transferable best principles and best practices to be gleaned from our experience in implementing and evaluating the ElderSmile program.

\section{Interpretation and Implications of the Findings}

Urban planners and public health practitioners are increasingly as focused on the social aspects of neighborhood environments as they are on the physical ones, including meeting places. ${ }^{37}$ In New York City, nearly one third $(31.3 \%)$ of older adults of all ages and of both genders living in NYCHA housing who responded to a telephone survey reported substantial use of senior centers, i.e., a few days a week or every day. ${ }^{38}$ In a representative sample of adults aged 60 years and older who attended a random selection of 56 senior centers in New York City, significant differences were found by race/ethnicity in factors related to oral health. ${ }^{39}$ In our study, we included center staff and caregivers accompanying older adults if they opted to participate in the ElderSmile program.

Beyond documenting attendance and need, investigators have also conducted research into implementing programs at senior centers and other community sites that address pain, ${ }^{40,41}$ oral health, ${ }^{42,}{ }^{43}$ stroke risk, ${ }^{44}$ and fall prevention. ${ }^{45,} 46$ Third places may also be considered as anchor institutions within poor neighborhoods that foster health equity (engagement, available resources, and information access $).{ }^{47}$

\section{CONCLUSION}

This study adds to the evidence based on evaluating place-based programs for health promotion by using the CFIR to guide program implementation and evaluation and introducing a locally relevant system for classifying center types and incorporating a spatial approach that provides insight into where participants live and how far they travel to attend outreach events at particular centers and types. We agree with Dunn that in the absence of real socioeconomic change for disadvantaged populations (external policy under outer setting), we may continue to be disappointed in the outcomes of place-based interventions. ${ }^{48}$ Nonetheless, action needs to occur on many levels simultaneously, and programs that reach older adults where they congregate in neighborhoods, i.e., third places, hold promise for intervening before health conditions become severe.

\section{ACKNOWLEDGMENTS}

The authors were supported in the research, analysis, and writing of this paper by the National Institute for Dental and Craniofacial Research and the Office of Behavioral and Social Sciences Research of the US National Institutes of Health for the projects titled, Leveraging Opportunities to Improve Oral Health in Older Adults (grant R21-DE021187) and Integrating Social and Systems Science Approaches to Promote Oral Health Equity (grant R01-DE023072). We thank 
Leydis De La Cruz and Rogelina Peralta for the program support toward the conduct of this study.

Open Access This article is distributed under the terms of the Creative Commons Attribution 4.0 International License (http://creativecommons.org/ licenses/by/4.0/), which permits unrestricted use, distribution, and reproduction in any medium, provided you give appropriate credit to the original author(s) and the source, provide a link to the Creative Commons license, and indicate if changes were made.

\section{APPENDIX}

TABLE 2 Consolidated Framework for Implementation Research (CFIR) constructs (adapted from the CFIR Research Team ${ }^{13}$ )

\begin{tabular}{|c|c|c|}
\hline \multicolumn{2}{|c|}{ Construct } & Short description \\
\hline \multicolumn{3}{|c|}{ I. Intervention characteristics } \\
\hline A & Intervention source & $\begin{array}{l}\text { Perception of key stakeholders about whether the intervention } \\
\text { is externally or internally developed }\end{array}$ \\
\hline B & Evidence strength & $\begin{array}{l}\text { Stakeholders' perceptions of the strength of evidence that the } \\
\text { intervention will have desired outcomes }\end{array}$ \\
\hline $\mathrm{C}$ & Relative advantage & $\begin{array}{l}\text { Stakeholders' perception of the advantage of implementing } \\
\text { the intervention versus an alternative solution }\end{array}$ \\
\hline D & Adaptability & $\begin{array}{l}\text { The degree to which an intervention can be adapted, tailored, } \\
\text { refined, or reinvented to meet local needs }\end{array}$ \\
\hline E & Trialability & $\begin{array}{l}\text { The ability to test the intervention on a small scale in the } \\
\text { organization and to be able to reverse course if warranted }\end{array}$ \\
\hline $\mathrm{F}$ & Complexity & $\begin{array}{l}\text { Perceived difficulty of implementation, reflected by duration, } \\
\text { scope, radicalness, disruptiveness, and intricacy }\end{array}$ \\
\hline G & Design packaging & $\begin{array}{l}\text { Perceived excellence in how the intervention is bundled, } \\
\text { presented, and assembled }\end{array}$ \\
\hline $\mathrm{H}$ & Costs & $\begin{array}{l}\text { Costs of the intervention and costs associated with } \\
\text { implementation including investment, supply, and } \\
\text { opportunity costs }\end{array}$ \\
\hline
\end{tabular}

II. Outer setting

A Patient needs

B Cosmopolitanism

C Peer pressure

D External policy

III. Inner setting

A Structure

B Networks

The extent to which patient needs, as well as barriers and facilitators, are accurately known and prioritized

The degree to which an organization is networked with other external organizations

Competitive pressure to implement an intervention, typically because key peer organizations have implemented it

A broad construct that includes external strategies to spread interventions, including policy and regulations

The social architecture, age, maturity, and size of an organization

The nature and quality of webs of social networks and the 


\section{Continued}

\section{Construct}

C Culture

D Climate

1 Tension for change

2 Compatibility

3 Relative priority

$4 \quad$ Incentives

$5 \quad$ Goals and feedback

6 Learning climate

E Readiness

1 Engagement

2 Available resources

3 Information access

IV. Characteristics of individuals

A Knowledge and beliefs

B Self-efficacy

C Stage of change

D Identification

E Personal attributes

V. Process

A Planning

B Engaging

$1 \quad$ Opinion leaders

$2 \quad$ Leaders

3 Champions

$4 \quad$ Change agents

C Executing
Short description

nature and quality of formal and informal communications

Norms, values, and basic assumptions of a given organization

The absorptive capacity for change and shared receptivity of involved individuals to an intervention

The degree to which stakeholders perceive the current situation as intolerable or needing change

The degree of tangible fit between meaning and values attached to the intervention by involved individuals

Individuals' shared perception of the importance of the implementation within the organization

Extrinsic incentives such as goal-sharing awards, performance reviews, promotions, and raises in salary

The degree to which goals are communicated, acted upon, and fed back to staff and alignment of feedback with goals

A climate in which there is sufficient time and space for reflective thinking and evaluation

Tangible and immediate indicators of organizational commitment to its decision to implement an intervention

Commitment, involvement, and accountability of leaders and managers with the implementation

The level of resources dedicated for implementation and ongoing operations, including money, training, and time

Ease of access to digestible information about the intervention and how to incorporate it into work tasks

Individuals' attitudes toward and value placed on the intervention as well as familiarity with facts, truths, and principles

Individual belief in their own capabilities to execute courses of action to achieve implementation goals

Characterization of the phase an individual is in, as she progresses toward skilled, enthusiastic, and sustained use

A broad construct related to how individuals perceive the organization and their degree of commitment

A broad construct to include personal traits such as tolerance of ambiguity, intellectual ability, and learning style

The degree to which a scheme and tasks for implementing an intervention are developed in advance

Attracting and involving appropriate individuals in the implementation and use of the intervention

Individuals in an organization who have formal or informal influence on the attitudes and beliefs of their colleagues

Individuals in the organization who have been formally appointed with responsibility for implementing an intervention

Individuals who dedicate themselves to supporting, marketing, and driving through an implementation

Individuals who are affiliated with an outside entity who formally influence intervention decisions in a desirable way

Carrying out or accomplishing the implementation according 


\section{Continued}

\begin{tabular}{ll}
\hline Construct & $\begin{array}{l}\text { Short description } \\
\text { Deflecting }\end{array} \quad \begin{array}{l}\text { to plan } \\
\text { Quantitative and qualitative feedback about the progress of } \\
\text { implementation accompanied with regular debriefing }\end{array}$ \\
\hline
\end{tabular}

\section{REFERENCES}

1. Oldenburg R. The great good place: cafes, coffee shops, bookstores, bars, hair salons, and other hangouts at the heart of a community. Saint Paul, MN: Paragon House; 1989. ISBN 1557781109.

2. Oldenburg R, ed. Celebrating the third place: inspiring stories about the "great good places" at the heart of our communities. New York, NY: Marlowe \& Company; 2001. ISBN 1-56924-612-2.

3. Frumkin H. Healthy places: exploring the evidence. Am J Public Health. 2003; 93(9): 1451-6. doi:10.2105/AJPH.93.9.1451.

4. Kaplan S, Kaplan R. Health, supportive environments, and the reasonable person model. Am J Public Health. 2003; 93(9): 1484-9. doi:10.2105/AJPH.93.9.1484.

5. Glover TD, Parry DC. A third place in the everyday lives of people living with cancer: functions of Gilda's Club of Greater Toronto. Health Place. 2009; 15(1): 97-106. doi:10. 1016/j.healthplace.2008.02.007.

6. Hooper CM, Ivory VC, Fougere G. Childhood neighbourhoods as third places: developing durable skills and preferences that enhance wellbeing in adulthood. Health Place. 2015; 34: 34-45. doi:10.1016/j.healthplace.2015.03.017.

7. Northridge ME, Nye A, Zhang YV, Jack G, Cohall AT. “Third places” for healthy aging: online opportunities for health promotion and disease management in adults in Harlem. $J$ Am Geriatr Soc. 2011; 59(1): 175-6. doi:10.1111/j.1532-5415.2010.03217.x.

8. Baum F, Palmer C. "Opportunity structures": urban landscape, social capital and health promotion in Australia. Health Promot Int. 2002; 17(4): 351-61. doi:10.1093/heapro/ 17.4.351.

9. Marshall S, Northridge ME, De La Cruz LD, Vaughan RD, O’Neil-Dunne J, Lamster IB. ElderSmile: a comprehensive approach to improving oral health for seniors. Am J Public Health. 2009; 99(4): 595-9. doi:10.2105/AJPH.2008.149211.

10. Northridge ME, Ue FV, Borrell LN, et al. Tooth loss and dental caries in communitydwelling older adults in northern Manhattan. Gerodontology. 2012; 29(2): e464-73. doi:10.1111/j.1741-2358.2011.00502.x.

11. Marshall SE, Cheng B, Northridge ME, Kunzel C, Huang C, Lamster IB. Integrating oral and general health screening at senior centers for minority elders. Am J Public Health. 2013; 103(6): 1022-5. doi:10.2105/AJPH.2013.301259.

12. Damschroder LJ, Aron DC, Keith RE, Kirsh SR, Alexander JA, Lowery JC. Fostering implementation of health services research findings into practice: a consolidated framework for advancing implementation science. Implement Sci. 2009; 4:50. doi:10. 1186/1748-5908-4-50.

13. CFIR Research Team. CFIR Technical Assistance Website. Ann Arbor, MI: Center for Clinical Management Research. Available at: http://cfirguide.org/index.html. Accessed July 11, 2016.

14. Borrell LN, Northridge ME, Miller DB, et al. Oral health and health care for older adults: a spatial approach for addressing disparities and planning services. Spec Care Dent. 2006; 26(6): 252-6. doi:10.1111/j.1754-4505.2006.tb01663.x. 
15. Widener MJ, Metcalf SS, Northridge ME, Chakraborty B, Marshall SM, Lamster IB. Exploring the role of peer density in the self-reported oral health outcomes of older adults: a kernel density based approach. Health Place. 2012; 18(4): 782-8. doi:10.1016/j. healthplace.2012.04.004.

16. Widener MJ, Northridge ME, Chakraborty B, et al. Patterns of chronic conditions in older adults: exploratory spatial findings from the ElderSmile program. Am J Prev Med. 2014; 46(6): 643-8. doi:10.1016/j.amepre.2014.01.013.

17. Kum SS, Wang H, Wang P, et al. The ElderSmile TimeMap: benefits of connecting statistics with time and place. Am J Public Health. 2015; 105(9): 1748-50. doi:10.2105/ AJPH.2015.302730.

18. New York City Department for the Aging. Housing Resources. New York, NY: City of New York; 2016. Available at: http://www.nyc.gov/html/dfta/html/benefits/housing.shtml. Accessed April 4, 2016.

19. Health Advocates for Older People. Senior housing opportunities in the borough of Manhattan, New York, NY. 5th ed. New York, NY: Health Advocates for Older People; 2015. Available at: http://www.cidny.org/resources/Services/HAFOP-Senior\%20Housing\%20Guide.pdf. Accessed April 4, 2016.

20. Northridge ME, Chakraborty B, Kunzel C, Metcalf S, Marshall S, Lamster IB. What contributes to self-rated oral health among community-dwelling older adults? Findings from the ElderSmile program. J Public Health Dent. 2012; 72(3): 235-45. doi:10.1111/j.1752-7325.2012.00313.x.

21. Northridge ME, Yu C, Chakraborty B, et al. A community-based oral public health approach to promote health equity. Am J Public Health. 2015; 105(Suppl 3): S459-65. doi:10.2105/AJPH. 2015.302562.

22. Marshall S, Schrimshaw EW, Metcalf SS, et al. Evidence from ElderSmile for diabetes and hypertension screening in oral health programs. J Calif Dent Assoc. 2015; 43(7): 379-87. PMCID: PMC4594879.

23. New York State Office for the Aging. Naturally Occurring Retirement Community Supportive Service Program (NORC-SSP) and Neighborhood NORC (NNORC). Albany, NY: New York State Office for the Aging; 2016. Available at: http://www.aging.ny.gov/NYSOFA/Programs/ CommunityBased/NORC-NNORC.cfm. Accessed March 2, 2016.

24. New York City Department for the Aging. NORC programs. New York, NY: New York City Department for the Aging; 2016. Available at: http://www.nyc.gov/html/dfta/html/services/ retirement.shtml. Accessed March 2, 2016.

25. US Department of Housing and Urban Development. Section 202 Supportive Housing for the Elderly Program. Washington, DC: US Department of Housing and Urban Development; 2016. Available at: http://portal.hud.gov/hudportal/HUD?src=/program_ offices/housing/mfh/progdesc/eld202. Accessed March 2, 2016.

26. New York City Housing Authority. Community Centers and Senior Centers. New York, NY: City of New York; 2016. Available at: www.nyc.gov/nycha. Accessed March 2, 2016.

27. New York Academy of Medicine. NYC Senior Centers: visioning the future. New York, NY: New York Academy of Medicine; 2010. Available at: http://www.cardi.ie/userfiles/ NYAM-NYCSeniorCentersfinal.pdf. Accessed April 7, 2016.

28. Griffin SO, Jones JA, Brunson D, Griffin PM, Bailey WD. Burden of oral disease among older adults and implications for public health priorities. Am J Public Health. 2012; 102(3): 411-8. doi:10.2105/AJPH.2011.300362.

29. Siemens. DCA Vantage Analyzer; 2014. Available at: http://www.healthcare.siemens.com/ point-of-care/diabetes/dca-vantage-analyzer. Accessed April 7, 2016.

30. American Diabetes Association. Classification and diagnosis of diabetes. Diabetes Care. 2015; 38(Suppl): S8-16. doi:10.2337/dc16-S005.

31. Omron Healthcare, Inc. Blood pressure monitors. Available at: https://omronhealthcare. com/blood-pressure/. Accessed April 28, 2016.

32. Centers for Disease Control and Prevention. High blood pressure facts. 2012. Available at: http://www.cdc.gov/bloodpressure/facts.htm. Accessed April 7, 2012.

33. R. Version 3.2.2. Vienna, Austria: R Foundation for Statistical Computing; 2015. 
34. Fleiss JL, Levin B, Paik MC. Statistical methods for rates and proportions. 3rd ed. Hoboken, NJ: John Wiley \& Sons; 2003.

35. New York City Department of City Planning. Geosupport Desktop Edition. Software version 16.1. New York, NY: City of New York. Available at: http:/www1.nyc.gov/site/planning/data-maps/ open-data/dwn-gde-home.page. Accessed April 7, 2016.

36. Desktop AGIS. Version 10.3. Redlands, CA: Environmental Systems Research Institute; 2014.

37. Macintyre S, Ellaway A, Cummins S. Place effects on health: how can we conceptualise, operationalise and measure them? Soc Sci Med. 2002; 55(1): 125-39.

38. Schneider AE, Ralph N, Olson C, Flatley AM, Thorpe L. Predictors of senior center use among older adults in New York City public housing. J Urban Health. 2014; 91(6): 1033-47. doi:10.1007/s11524-014-9906-3.

39. Shelley D, Russell S, Parikh NS, Fahs M. Ethnic disparities in self-reported health status and access to care among older adults in NYC. J Urban Health. 2011; 88(4): 651-62. doi:10.1007/s11524-011-9555-8.

40. Townley S, Papaleontiou M, Amanfo L, et al. Preparing to implement a self-management program for back pain in New York City senior centers: what do prospective consumers think? Pain Med. 2010; 11(3): 405-15. doi:10.1111/j.1526-4637.2009.00783.x.

41. Tobias KR, Lama SD, Parker SJ, Henderson CR Jr, Nickerson AJ, Reid MC. Meeting the public health challenge of pain in later life: what role can senior centers play? Pain Manag Nurs. 2014; 15(4): 760-7. doi:10.1016/j.pmn.2013.07.013.

42. Glassman P, Harrington M, Mertz E, Namakian M. The virtual dental home: implications for policy and strategy. J Calif Dent Assoc. 2012; 40(7): 605-11. PMCID:PMC3477859.

43. Northridge ME, Shedlin M, Schrimshaw EW, et al. Recruitment of racial/ethnic minority older adults through community sites for focus group discussions. BMC Public Health. (in review)

44. Kwon I, Choi S, Mittman B, et al. Study protocol of "Worth the Walk": a randomized controlled trial of a stroke risk reduction walking intervention among racial/ethnic minority older adults with hypertension in community senior centers. BMC Neurol. 2015; 15: 91. doi:10.1186/s12883-015-0346-9.

45. Baker DI, Gottschalk M, Bianco LM. Step by step: integrating evidence-based fall-risk management into senior centers. Gerontologist. 2007; 47(4): 548-54.

46. Li F, Harmer P. Protocol for disseminating an evidence-based fall prevention program in community senior centers: evaluation of translatability and public health impact via a single group pre-post study. Implement Sci. 2014; 9: 63. doi:10.1186/1748-5908-9-63.

47. Smedley B, Amaro H. Advancing the science and practice of place-based intervention. Am J Public Health. 2016; 106(2): 197. doi:10.2105/AJPH.2015.303043.

48. Dunn JR. Evaluating place-based programmes for health improvement. J Epidemiol Community Health. 2014; 68(7): 591. doi:10.1136/jech-2014-204431. 